Mots. Les langages du politique

Discours d'autorité : des discours sans éclat(s)?

\title{
Un effacement énonciatif paradoxal. Les discours des grands leaders républicains sur le suffrage universel
}

A paradoxal enunciative fading off. The speeches of the main leaders of the Republic about the universal suffrage

Un desaparecimiento enunciativo paradójico. Los discursos de los grandes

líderes republicanos sobre el sufragio universal

\section{Aude Dontenwille-Gerbaud}

\section{OpenEdition}

\section{Journals}

Édition électronique

URL : https://journals.openedition.org/mots/21862

DOI : $10.4000 /$ mots. 21862

ISSN : 1960-6001

Éditeur

ENS Éditions

Édition imprimée

Date de publication : 23 mars 2015

Pagination : 35-47

ISBN : 978-2-84788-698-6

ISSN : 0243-6450

\section{Référence électronique}

Aude Dontenwille-Gerbaud, « Un effacement énonciatif paradoxal. Les discours des grands leaders républicains sur le suffrage universel », Mots. Les langages du politique [En ligne], 107 | 2015, mis en ligne le 23 mars 2017, consulté le 23 avril 2022. URL : http://journals.openedition.org/mots/21862 ; DOI : https://doi.org/10.4000/mots.21862 


\section{Un effacement énonciatif paradoxal. Les discours des grands leaders républicains sur le suffrage universel}

Si l'on considère les dix premières années de fondation de la Troisième République et les discours des grands leaders qui s'expriment devant différents publics extraparlementaires, le suffrage universel apparaît comme une évidence quasi sacralisée. II ne se discute pas, ne s'argumente pas. Il est le droit par excellence d'une «forme qui emporte et résout le fond », selon l'expression de Léon Gambetta pour désigner la République. Tous les débats autour du bienfondé du suffrage universel sont tout simplement passés sous silence, que ce soit les revendications pour des candidatures ouvrières, celles des intellectuels positivistes qui considèrent que le suffrage universel est une prise de risque trop importante pour la démocratie, celles des conservateurs qui refusent l'expression de la «vile multitude » ou encore les quelques rares voix féminines qui s'insurgent contre l'universalité présumée d'un suffrage qui les exclut.

Ce ne sont plus Léon Gambetta, Jules Ferry, Victor Hugo, Paul Bert qui s'expriment, mais un « instinct», une « voix profonde de la France » dont ils ne sont plus qu'un écho intemporel. L'ethos de ces grands leaders s'efface dans une forme d'interaction orateur/public qui s'apparente à une grand-messe républicaine. Cet article cherchera à montrer en quoi ces discours peuvent être qualifiés de discours d'autorité. Comment, au sein de discours qui peuvent parfois durer près de deux heures, s'opère ce changement discursif entre la rhétorique classique de l'idée républicaine et un discours d'autorité sur le suffrage universel? Qu'apporte ce discours d'autorité dans une perspective d'efficacité, quel est son statut pragmatique? Enfin, quels effets perlocutoires de ce discours d'autorité peut-on apprécier dans la réception de cet acte de langage? Quelle trace peut-on en trouver au sein des discours et comment confronter cette trace avec celle laissée par les sténographes de l'époque qui indiquent les réactions des publics présents?

Nous partirons d'un corpus de 164 discours, ceux des grands leaders devant des publics extraparlementaires, dont la retranscription disponible prend en 
compte les réactions des publics ${ }^{1}$. Ils sont datés de 1871 (quelques mois après la proclamation de la République) à 1882 (mort de Léon Gambetta et recomposition des forces politiques). Nous en choisirons ici quatre extraits, discours tenus par des orateurs particulièrement emblématiques de ces grandes voix de l'époque. Nous étudierons ainsi l'acte discursif fondateur de la Troisième République en présentant dans un premier temps l'expression d'un malaise à l'égard du suffrage universel, puis un processus d'effacement énonciatif et enfin l'interaction entre orateur et public dans la prise en charge de ce discours d'autorité.

\section{L'expression d'un malaise}

Dans une lettre de décembre 1851, quelques jours après le résultat du plébiscite qui approuve à une très large majorité le coup d’État de Napoléon III, George Sand évoque son désespoir à son ami, l'imprimeur P.-J. Hetzel :

Que pouvons-nous répondre à 6 ou 7 millions de votes? Ils sont un peu extorqués, il est vrai, le vote sans le droit de choisir, sans le droit de se réunir, de se consulter et de s'entendre, c'est bien un escamotage. Mais, après tout, personne n'était obligé de dire OUI et la grande majorité a dit OUI. On n'achète pas et on n'intimide pas 6 à 7 millions d'hommes; hélas! la France n'est pas républicaine, elle ne l'a jamais été. (Parménie, Bonnier de La Chapelle, 1985, p. 158)

Se trouve posée dans ces quelques lignes une question qui va hanter les républicains jusqu'aux élections de 1879 , lesquelles verront enfin une majorité républicaine s'installer au Parlement. Le suffrage universel fait peur. Philosophiquement, certains comme Charles Renouvier vont dialoguer avec Kant pour interroger ce dilemme. En effet, dans son ouvrage La Religion dans les limites de la raison, Kant refuse l'idée même qu'un peuple puisse ne pas être mûr pour la liberté :

J'avoue que je ne puis admettre le langage de la prudence, lorsqu'elle dit : «tel peuple n'est pas mûr pour la liberté». Avec pareille supposition, on n'entrerait jamais dans la liberté, car on ne peut mûrir pour elle avant d'être placé dans son sein même : il faut être libre pour pouvoir se servir, conformément à son but, des forces que donne la liberté [...] Je n'ai rien à dire contre ceux qui ont le pouvoir en main et qui, forcés par les circonstances, ajournent même fort loin l'époque où les chaînes seront brisées. Mais poser en principe que ceux que l'on tient sous sa domination ne sont point, en général, faits pour la liberté, et que l'on a le droit de les en tenir éloignés à jamais, c'est empiéter sur les droits de Dieu, qui a fait l'homme pour la liberté. (Kant, 1841, p. 344)

Cette tension entre liberté et maturité se retrouve dans une argumentation républicaine paradoxale. Marie-Claude Blais, analysant l'œuvre entière du phi-

1. Voir corpus en fin d'article. 
losophe kantien Charles Renouvier, montre comment l'incertitude inhérente à l'appréciation du possible n'empêche pas la certitude sur le souhaitable (Blais, 2000, p. 265). L'horizon d'attente (Koselleck, 1990) des républicains reste bien celui d'une République Une et Indivisible pour un peuple de citoyens libres. La rencontre entre ces orateurs et leurs publics participe de l'événement discursif (Guilhaumou, 2006) fondateur de la Troisième République, véritable champ d'expérimentation : se montrer sage, homme régénéré donc libre, digne du suffrage universel, tout en héritant du bouillonnement des actes fondateurs de la Révolution. Ces temps de rencontre se veulent formation conjointe, expérience vécue d'une relation entre citoyens et représentants. Rien n'oblige ces orateurs à venir s'exprimer devant ces publics déjà acquis à l'idée républicaine, sauf à accepter que la République ne saurait se fonder dans les seules instances parlementaires. L'enjeu de l'interaction orateur/public n'est autre que celui de la liberté en acte, une liberté qui fait peur à nombre de républicains. Comment se déploie cette tension au sein du discours des orateurs qui s'expriment devant des publics extraparlementaires, pour l'essentiel populaires ${ }^{2}$ ?

Si nous considérons l'ensemble de notre corpus, alors même que la question du suffrage universel est au cœur de toutes les préoccupations, et notamment lors des multiples consultations électorales, il semble que les orateurs des débuts de la Troisième République hésitent beaucoup à employer le terme. Nul, parmi eux, ne remet en cause le suffrage universel. Mais le malaise ne permet plus de l'évoquer facilement. Une simple recherche des occurrences montre qu'au sein de notre corpus, seuls $71 \%$ des discours utilisent le terme. Ceux de Léon Gambetta se situent dans cette moyenne. Comparativement, les syntagmes république, France, liberté, nation se retrouvent dans la presque totalité de ses discours. Manifestement, l'orateur parle toujours du suffrage universel sans toutefois oser le nommer, alors même que la pratique des réunions, le choix de ces grands moments de rencontre avec les publics, n'ont d'autre but, à ses yeux, que de former des citoyens éclairés. Paul Bert et Jules Ferry paraissent encore plus embarrassés puisque $54 \%$ des discours pour l'un, et même seulement $28 \%$ pour l'autre citent le syntagme suffrage universel. Faut-il y voir un effet de génération? Victor Hugo ou Charles Floquet emploient quant à eux ce terme dans $88 \%$ de leurs discours, comme si les vétérans de 1848 revendiquaient hautement l'expérience fondatrice de la Seconde République. S'agit-il d'un implicite pour la génération nouvelle, un «cela va de soi » de l'argumentation qui échapperait ainsi à toute contestation (Amossy, 2000)? L'argumentation insiste toutefois sur l'éducation nécessaire du peuple, sur la question de son expérience. Le suffrage universel ne va donc pas de soi. Il est

2. Les indications sur les comités organisateurs de ces rencontres, les articles de presse qui couvrent ces «événements », les correspondances privées permettent de qualifier une bonne partie de ces publics de «populaires». Voir Dontenwille-Gerbaud, à paraître. 
dès lors possible d'évoquer une nouvelle hypothèse : la stratégie discursive de sous-emploi du syntagme suffrage universel obéirait à d'autres logiques, en particulier à une logique d'euphémisation. Ainsi de cette citation de Jules Ferry :

Le suffrage universel n'est pas seulement une institution sacrée et souveraine, c'est toute une politique et presque un symbole. II n'est pas seulement le fait, le Droit, le Juste, il est aussi l'Inévitable. Il est tout le présent et il est tout l'avenir. Le suffrage universel est l'honneur des multitudes, le gage des déshérités, la réconciliation des classes, la vie légale pour tous. C'est en lui seul qu'il faut désormais vivre, espérer et croire. Même ennemi, il faut l'aimer. (Jules Ferry, 1893, p. 92)

Ce texte date de 1863, époque du Second Empire. Jules Ferry défend ici l'idée de candidatures au Corps législatif qui ne soient pas «officielles», en d'autres termes la possibilité de candidatures d'opposition républicaine. L'interdiscours est celui d'un débat très vif sur l'opportunité de proposer ou non des candidatures ouvrières. Le Manifeste des Soixante qui milite pour ces candidatures ne fait pas l'unanimité parmi les républicains. L'argument de nombreux républicains consiste à opposer à ces candidatures ouvrières la nation Une et Indivisible : la catégorie sociale de l'élu ne doit pas entrer en ligne de compte. Si la France forme Un peuple, l'intelligence se doit d'être partagée par tous et non par une élite, fût-elle ouvrière. Ces quelques lignes de Jules Ferry datées de 1863 peuvent s'apparenter à une véritable «langue de bois» (Delporte, 2009, p. 10) : aucune information vérifiable, pas d'argument susceptible d'être contredit, des euphémismes et des mots chocs pour une réalité bien difficile à conceptualiser... Si l'on définit l'euphémisme comme un détour par rapport à un contenu immédiat (Jamet, Jobert, 2010), l'expression « réconciliation des classes » forme bien un détour pour ne pas dire la fracture de juin 1848 entre républicains bourgeois et monde ouvrier, et pour taire le débat sur les candidatures ouvrières. "La vie légale pour tous» est un autre contour pour ne pas dire le combat de certaines femmes qui revendiquent le suffrage réellement universel. Qui dit « réconciliation » évoque implicitement un « combat». La stratégie énonciative va donc dans le sens de l'atténuation du potentiel menaçant que constituent les référents problématiques : multitude, déshérités, classes. Les combats républicains sont discursivement estompés au travers de cette euphémisation, même s'ils sont désignés en creux (Bonhomme, 2005, p. 243). L'accumulation de termes positifs (honneur, gage, vie légale) opère une véritable réévaluation axiologique des combats républicains. Le discours d'autorité se fait ici acte de foi, au-delà donc de toute argumentation : le Droit, le Juste, l'Inévitable, soulignés typographiquement par des majuscules. Il convient «d'espérer, de croire».

Pour Pierre Rosanvallon (1992, p. 341), « le suffrage universel constitue l'horizon indépassable de l'idée républicaine». Mais c'est pour interroger aussitôt cette idée républicaine : est-elle philosophiquement démocratique? Tout concourt, d'après l'analyse proposée par Pierre Rosanvallon, à soulever le pro- 
blème de la place des élites : devant l'ingratitude des masses, aucune théorie de la pratique démocratique ne voit le jour. La ferveur démopédique 3 des années 1880 s'explique par le besoin de réduire l'écart entre la logique du suffrage universel et les impératifs de la raison. Le suffrage-souveraineté fonde la République en droit. La focalisation sur l'immaturité du peuple permet au suffrage-appartenance de n'être pas interrogeable. Les conservateurs républicains utilisent le suffrage universel comme un moyen de clore le champ de la réflexion politique et intellectuelle en même temps que celui de la guerre sociale.

Il est toutefois une autre façon de poser le problème. Philippe Schepens, relisant Paul Ricoeur, s'interroge sur le hiatus continuel constitutif du lien entre idéologie et utopie, hiatus entre le discours de légitimation que le groupe dominant défend et la manière dont les citoyens l'investissent, ou entre «les prétentions de l'autorité » et « la croyance des citoyens ». Pour Paul Ricoeur, il n'existe pas de "système absolument rationnel de légitimité » (Schepens, 2011, p. 32). L'analyse de l'interaction entre orateurs et public permet-elle de voir émerger cette tension, ce hiatus entre discours de légitimation et croyances des citoyens? Nous gardons présent à l'esprit que les réactions notées par les sténographes (qu'il convient de ne pas essentialiser) ne sont qu'une réécriture qui ne permettra jamais que d'évoquer quelques pistes d'analyse par comparaison d'un discours à l'autre.

\section{L'effacement énonciatif d'une voix tonitruante...}

Léon Gambetta représente le tribun par excellence, la voix tonitruante de la République depuis 1868 , celle que la presse admire, critique, caricature à souhait. Nous avons déjà analysé l'argument d'autorité que constitue le suffrage universel dans un discours de Léon Gambetta à propos d'une bataille de chiffres (Dontenwille-Gerbaud, 2012, p.46). L'orateur y assoit alors sa légitimité de leader sur l'autorité du résultat électoral. Mais tout autre peut être parfois sa stratégie discursive.

En avril 1881, Gambetta est président de la Chambre des députés. Il prend la parole lors d'un banquet devant les membres de la Ligue de l'enseignement. L'ethos préalable est celui tout à la fois d'un homme d'État et d'un militant de longue date, dans un croisement entre discours politique et discours institutionnel. «Il y a un mot dans votre discours, Monsieur Macé, que je vous demande la permission de reprendre» (Gambetta, 1881, vol. IX, p. 199). La scène de parole (Maingueneau, 2002, p.515) instituée par cette énonciation

3. Pour les républicains, la question de l'École est décisive et ne se réduit pas à la «question scolaire». Pour que le peuple soit souverain, il faut qu'il soit éduqué. Il faut former des citoyens. Proudhon liait le règne du peuple, la démocratie au vrai sens du terme, à son éducation, qu'il appelait « démopédie». 
singulière est celle d'un grand qui reconnaît son aîné, un maître, un vieux militant de l'instruction populaire à qui l'on doit le respect. «Je vous demande la permission... », "Permettez-moi de dire qu'en vous entendant, il me semblait... ». L'ethos discursif se détache de l'ethos préalable par une minimisation du poids de l'orateur. Le discours se poursuit par un argument que l'on peut qualifier d'autorité, souligné par un acquiescement du public présent :

Vous avez dit que la pensée première de votre œuvre vous avait été suggérée dès 1866, par l'existence du suffrage universel parmi nous et par le spectacle de ses défaillances et de ses chutes. C'est alors, disiez-vous, que vous aviez réclamé l'instruction universelle comme un antidote du suffrage universel. Eh bien, permettezmoi de dire qu'en vous entendant, il me semblait que vous alliez peut-être dépasser la légitime mesure, car le suffrage universel est un droit avant d'être l'exercice légal et régulier de la raison cultivée. (C'est vrai ! Très bien! Très bien!) (Gambetta, 1881, ibid.)

Il ne s'agit pas ici d'une simple manifestation d'autorité s'appuyant sur la force de l'assertion. Ce n'est pas un homme qui juge et affirme, mais un locuteur second, qui n'est autre que la constitution républicaine, traduction institutionnelle du projet républicain énoncé depuis 1848 .

Mais le discours d'autorité se défait. Léon Gambetta commence par essentialiser le suffrage universel qui devient lui-même acteur, dans un mécanisme de concession qui prend en charge l'interdiscours où se déploient les inquiétudes :

Sans doute le suffrage universel a pu fléchir et même succomber; il a pu être entraîné, surpris; il a pu être sophistiqué; sans doute, comme l'humanité ellemême dans sa marche incessante vers le progrès, il a pu avoir ses éclipses et ses défaillances, mais il a eu aussi ses grandeurs et n'oublions jamais que ce n'est qu'en s'éprouvant lui-même à la pierre de touche de l'expérience qu'il peut prendre complètement et toute sa compétence et toute son autorité. (Ibid.)

Répété quatre fois, le segment «il a pu» rend compte de l'expérience douloureuse des républicains. Puis le locuteur se détache de l'énonciateur : ce n'est plus Léon Gambetta qui parle, mais Auguste Comte. « N'oublions jamais» s'adresse à un auditoire très largement acquis aux pensées positivistes sur l'expérience en politique et le rôle de l'opinion. Rien, toutefois, ne vient ici étayer l'argumentaire d'une expérience en elle-même fondatrice. Nous sommes en pleine doxa.

Oui, le suffrage universel est le droit; il est le droit en exercice et il ne faudrait pas laisser dire un seul instant que son principe ou sa valeur peuvent dépendre de l'état intellectuel de tout un peuple, car cet état intellectuel, nul n'est en possession de le mesurer. (Ibid.)

Cette fois, c'est Kant qui parle. La contradiction philosophique avec la phrase précédente est manifeste. Une pensée de la liberté et de la responsabi- 
lité de l'individu ne peut coexister avec une pensée positiviste sur les «Dignes prolétaires ", élite d'une nation seule capable d'entraîner le collectif. Comment, sur un socle idéaliste, construire une méthode de progrès raisonné positiviste? Pour les philosophes contemporains de Léon Gambetta, la pensée de l'orateur n'est pas facile à classer :

Quelles étaient au juste les opinions philosophiques de Léon Gambetta? Certaines affirmations récentes mettent la question à l'ordre du jour. Était-il attaché à une École, à un maître, comme on l'a prétendu après qu'il eut, par deux fois, en des occasions solennelles rendu hommage au «Positivisme » et magnifié le nom d'Auguste Comte qu'il saluait comme «le plus grand penseur du siècle »? Et s'il n'était pas positiviste, avait-il une doctrine métaphysique? [...] Était-il un pur idéaliste, un spiritualiste ou un matérialiste, un déiste, un athée ou un panthéiste? Si, pour trouver réponse à tant de points d'interrogation, on consulte ses discours ou ses écrits, on s'étonnera peut-être de n'y trouver nul indice d'une affirmation ou d'une négation doctrinale (Deluns-Montaud, 1897, p. 241)

Le discours d'autorité sur le suffrage universel tenu par Léon Gambetta n'est donc pas théoriquement fondé, il est même contradictoire. Discursivement, il n'est possible que par l'effacement du locuteur qui se retire en quelque sorte de l'énonciation. Il y a gommage des marques de sa présence au profit du «il», suffrage universel essentialisé, dans un interdiscours où dialoguent entre eux Kant et Auguste Comte. Le «je » du locuteur-énonciateur disparaît (Rabatel, 2004, p.3).

Qu'en est-il de la réception de ce discours? Les marques de réaction du public notées par le sténographe permettent d'en avoir quelques indices, même s'il s'agit bien évidemment d'une interprétation. Salle du Trocadéro, ce 21 avril 1881, le public apparaît instantanément en interaction avec l'orateur. Lorsque celui-ci débute son discours par une apologie de Jean Macé, fondateur de la Ligue de l'enseignement, les «Très bien!», les «Oui! », les «vifs applaudissements » éclatent de toute part. Mais lorsque Léon Gambetta semble faire un reproche à Jean Macé, celui de douter du suffrage universel, le sténographe ne note aucune réaction. Ensuite, le discours se poursuit par l'analyse d'un mot de Proudhon, celui de «démopédie». Le silence, aux dires du sténographe, semble s'installer durablement. Il est certes difficile d'interpréter les silences (Dayan, 1992) mais il est possible, par comparaison, de constater qu'il faudra à Gambetta utiliser une rhétorique bien rodée sur les vertus de l'école et les bienfaits de l'instituteur pour rétablir un contact direct avec le public. Il semble y réussir, puisque la fin du discours voit celui-ci réagir à nouveau avec vigueur. Comment interpréter ce silence dont rend compte le sténographe? Une première analyse consiste à considérer un public en désaccord avec l'orateur. Auditoire de la Ligue de l'enseignement, ce public n'accepte peut-être pas les critiques de Gambetta à propos de Jean Macé dont il partage certainement les inquiétudes à propos des «erreurs du suffrage universel». Une autre analyse, 
toutefois, est possible : à l'effacement énonciatif du locuteur-orateur correspond l'effacement des réactions du public. Gambetta est un tribun admiré et applaudi lorsqu'il se donne à voir. L'effacement de Gambetta, au profit d'un suffrage universel essentialisé, puis d'un double discours philosophique kantien et positiviste, donc incohérent, peut laisser le public sans voix.

\section{La grand-messe républicaine?}

Tout autre semble être l'interaction entre Victor Hugo et son public, dans un discours d'autorité sur le suffrage universel tenu le 12 octobre 1877 dans le $9^{e}$ arrondissement en soutien à la candidature de Jules Grévy.

Ce que je veux affirmer, et affirmer inflexiblement, c'est le profond respect dû par le pouvoir à la loi, et au législateur qui fait la loi, et au suffrage universel qui fait le législateur.

Vous le voyez, Messieurs, d'échelon en échelon, c'est au suffrage universel qu'il faut remonter. Il est le point de départ et le point d'arrivée; il a le premier et le dernier mot.

Messieurs, le suffrage universel va parler, et ce qu'il dira sera souverain et définitif. La parole suprême que va prononcer l'auguste voix de la France sera à la fois un décret et un arrêt, décret pour la république, arrêt contre la monarchie. (Oui ! Oui ! Applaudissements.) (Victor Hugo, 1968, vol. IV, p.51)

L'ethos de grand exilé, de chantre de l'amnistie permet à Victor Hugo d'être incisif : «je veux affirmer, et affirmer inflexiblement». Le locuteur et l'énonciateur ne font qu'un dans un ethos discursif qui ne fait que reprendre l'ethos préalable. La scène d'énonciation est alors celle de l'orateur inscrit dans une histoire tout autant personnelle qu'historique. Dans «Vous le voyez, Messieurs», se donne à voir l'interaction avec le public qui est appelé de façon directive à suivre la rhétorique qui se déploie. Puis l'énonciateur se dissocie du locuteur, forme discursive soulignée par le métadiscours «le suffrage universel va parler». On n'est pas loin de la parole divine, de l'alpha et l'oméga du Verbe. L'hyperénonciateur qu'est le suffrage universel fait disparaître le locuteur-énonciateur. L'effacement de l'énonciateur se marque sur le plan discursif par la personnification du suffrage universel (la voix auguste de la France) à laquelle participent conjointement l'orateur et son public qui approuve et applaudit. Nous sommes là dans une forme d'interaction qui s'apparente à une véritable communion.

Paula Cossart analyse, à propos des meetings politiques de ces années de fondation républicaine, la contradiction entre un projet de formation d'un citoyen éclairé, capable de débattre, de réfléchir par lui-même, plein de «retenue démocratique», et les effets d'une rhétorique des passions, qui semblerait vouloir dire qu'un orateur habile et convaincant impose ses idées (Cossart, 2010, p. 191). Le projet affirmé des Pères fondateurs de la République n’est pas 
de refuser les élans collectifs, mais d'éduquer le public à n'ovationner que le principe, la République elle-même, en aucun cas l'orateur.

Discursivement, l'effacement énonciatif, la place des hyperénonciateurs confèrent au discours sur le suffrage universel une autorité quasi sacralisée. Le suffrage universel ne s'argumente pas. C'est aussi la limite de l'exercice politique. Si la grand-messe fonctionne durant les années de danger - celles où la République reste encore à fonder -, la faiblesse du discours d'autorité se donne à voir dans des interactions plus difficiles à partir des années 1880. À Coulanges par exemple, le 15 août 1880, le discours d'autorité de Paul Bert sur le suffrage universel semble laisser le public silencieux. «Mes chers concitoyens, c'est à titre de représentant de la Nation, titre que vos suffrages ont contribué à me faire obtenir, que j'ai l'honneur de présider ce banquet» (Bert, 1881, p. 444). L'ethos préalable d'homme politique se retravaille ici dans une scénographie où domine le respect : "vos suffrages ont contribué», «j'ai l'honneur de présider». L'orateur s'inscrit dans une relation directe avec son auditoire. Puis il modifie son ethos. Paul Bert n'est plus seulement un élu, mais le représentant d'une majorité politique, celle des républicains opportunistes, aux affaires.

[...] j'ai donc le droit et même le devoir de boire au gouvernement que la France s'est donné, parmi tant de difficultés, de résistances, d'angoisses et de périls, au gouvernement de la Nation maîtresse d'elle-même, triomphante et toute puissante, au gouvernement de la République française [...] Je bois à la République, non pour lui souhaiter une force, une vie qu'elle possède, ni un avenir et une prospérité dont elle est sûre, mais pour la saluer et l'honorer (Approbation). (Bert, 1881, ibid.)

Le terme gouvernement revient trois fois dans la même phrase, la répétition constituant un marquage discursif d'autorité. Le sténographe présent note une «approbation », mais pas d'explosion d'applaudissements, et ce malgré l'utilisation du registre du pathos (difficultés, résistances, angoisses, périls). Puis le «je» disparaît au profit du «nous»:

Et nous pouvons tous lui rendre hommage, mes chers concitoyens, républicains de la veille ou du lendemain, de sentiment ou de raison, de conviction ou de nécessité, républicains de bonne volonté ou républicains malgré eux, car il en est, je le sais, et ce ne sont pas toujours les plus mauvais ni les moins sûrs. Les premiers ont le droit de se réjouir de voir enfin réaliser leur rêve... [...] car c'est en grande partie à leur sagesse, à leur énergie, à leur patience, qu'est dû le succès.

Oui, sous la direction et à l'exemple de trois hommes éminents, dont nous devons, en toute circonstance, honorer les noms et le souvenir, de Thiers, de Grévy, de Gambetta, ils ont su calmer les défiances, rassurer les intérêts, se mettre aux affaires, devenir patients, joindre la pratique quotidienne à la théorie qui ne leur avait jamais manqué! (Ibid.)

L'énonciateur-locuteur se dissout dans une unité de groupe, groupe qui va faire ce geste de porter un toast, quelles que soient par ailleurs, les différences 
des uns et des autres sur l'échiquier politique du moment. La volonté affichée de réaliser l'union ne s'argumente pas.

Oui, les républicains de la dernière heure, d'aujourd'hui, de ce soir (Sourires) car chaque jour leur nombre augmente, ceux-là mêmes qui n'ont rallié les autres que contraints et forcés, rendent, en buvant à la République, hommage au seul gouvernement qui soit aujourd'hui possible, au seul qu'une majorité solide puisse acclamer dans la France du suffrage universel. Ils s'inclinent d'abord devant la puissance des faits accomplis, devant la fatalité des situations. (Ibid.)

Le recours, classique en rhétorique, à une captatio benevolentiae (les républicains malgré eux ne sont pas les plus mauvais, et les républicains de la première heure sont les héros du jour) fonctionne dans l'interaction doublement : non pas seulement pour capter un public, mais également pour unir les républicains. Ceux de la première heure qui écoutent le discours ne peuvent plus rendre hommage à la République sans y associer «les républicains malgré eux», vraisemblablement peu présents physiquement lors de ce banquet. Le discours se poursuit à la troisième personne: «ils ont su calmer les défiances, rassurer les intérêts, se mettre aux affaires, devenir patients, joindre la pratique quotidienne à la théorie qui ne leur avait jamais manqué ! „ L'univers diégétique du récit doit être interprétativement construit par ces républicains de la première heure, directement acteurs du récit proposé. Cette narration à la troisième personne (le style de l'histoire selon Benveniste) semble ne susciter aucune réaction si ce n'est un sourire noté par le sténographe lorsque l'orateur insiste : «les républicains de la dernière heure, d'aujourd'hui, de ce soir (Sourires)». L'orateur n'est pourtant pas dans le registre de l'ironie. Ce «ils» rend «hommage au seul gouvernement qui soit aujourd'hui possible, au seul qu'une majorité solide puisse acclamer dans la France du suffrage universel ». Le suffrage universel devient ici l'acteur même du destin. Alain Rabatel emprunte à Patrick Charaudeau l’idée de «simulacre énonciatif», comme si le sujet parlant disparaissait complètement dans l'acte d'énonciation et laissait le discours parler par lui-même (Rabatel, 2004). L'ethos de Paul Bert n'est plus celui d'un représentant, d'un homme politique. Paul Bert n'est plus que la voix du destin devant laquelle aucune argumentation n'est possible. Cette voix explique, rend compte de ce qui s'est passé. La force illocutoire du constat fait le discours d'autorité : il n'y a plus qu'à s'incliner devant la puissance des faits. Est-ce la raison du silence du public? Nous ne pourrons jamais qu'en formuler l'hypothèse.

Pour conclure, dans ces grand-messes républicaines, le suffrage universel tend à devenir un terme-notion, chargé d'une valeur axiologique répétée inlassablement. L'énonciateur n'est autre que l'histoire de France elle-même et les locuteurs, fussent-ils de célèbres et tempétueux orateurs, ne font plus que lui prêter leurs voix. De la même façon que l'ont montré Hugues Constantin de 
Chanay et Sylvianne Rémi-Giraud à propos du terme démocratie, le syntagme suffrage universel se trouve «mis en tension entre, d'une part, un devoir-faire qui le tire vers le monde virtuel des principes abstraits et, d'autre part, un faire qui l'inscrit dans l'actualité de l'expérience - ce qui peut conduire à un questionnement implicite sur la modalité du “savoir" (comment faire?) » (de Chanay, Rémi-Giraud, 2007, p. 82). Toutefois, dans les années 1870-1880, le suffrage universel n'est pas un passage argumentatif obligé, ou plutôt, il n'est obligé que dans le discours républicain, lui conférant alors une dimension de ralliement vers l'horizon d'attente d'une République Une et Indivisible encore refusée par une opposition royaliste et bonapartiste. Ces publics sont déjà convaincus; les orateurs n'ont pas à défendre l'idée même de République, mais à développer un argumentaire solide pour des messagers qui auront charge ensuite d'aller répandre la «bonne nouvelle». Or, le suffrage universel n'est pas argumenté. Les orateurs pourraient s'emparer d'enjeux constitutionnels pour travailler la question de la représentativité. Ils ne le font pas, préférant se situer dans un discours qui se suffit à lui-même. Par là même, ils refusent les débats de l'époque, nombreux et vifs, sur cette représentativité. L'analyse des réactions notées par les sténographes permet de formuler quelques hypothèses sur les modalités de l'interaction entre orateur et publics. Ces derniers ne semblent pas simplement «manipulés». Dans sa lecture de Paul Ricoeur, Philippe Schepens retient le lien entre idéologie et utopie :

[...] pour Ricœur, il n'y a donc pas de pure domination idéologique. Non seulement les sujets psychologiques et sociaux disposent toujours, dans les transactions sociales, de positions qui leur permettent d'apercevoir, même confusément, les principes possibles d'une critique de l'idéologie du pouvoir en place, d'une utopie même partielle. Mais de plus, ce n'est pas seulement une faille inscrite dans les rapports sociaux, c'est une fonction même de l'imaginaire, mais dans le lien que cette fonction établit avec la praxis et avec les cadres symboliques dont elle dispose. Certes les sujets peuvent faire l'objet de captations imaginaires dans des représentations idéologiques qui voilent et distordent le réel, servent et confortent des intérêts de classe, légitiment un pouvoir, mais c'est cette même fonction imaginaire qui, prenant appui sur les hiatus dynamiquement inscrits dans les rapports sociaux, les oppositions voire les contradictions de classes, permet de construire des représentations alternatives. «Les symboles qui règlent notre identité ne proviennent pas seulement de notre présent et de notre passé mais aussi de nos attentes à l'égard du futur» (p.408), dit encore Ricœur de manière suggestive. (Schepens, 2011, p. 24)

Si les publics populaires, notamment ouvriers, sont d'abord républicains dans les années 1870-1880, s'élabore en même temps une réflexion sur la question sociale. L'horizon d'attente d'une République Une et Indivisible se trouve de plus en plus ouvertement en concurrence avec celui d'une République démocratique et sociale. D’après les sténographes, les publics semblent souvent silencieux lors de l'évocation du suffrage universel, alors même qu'ils réagissent facilement à d'autres propos. Ces silences peuvent s'inscrire dans 
le hiatus conceptualisé par Paul Ricoeur, celui de la liberté intrinsèque des individus. Pour faire applaudir le suffrage universel, ce n'est pas un discours d'autorité qui est nécessaire, mais un discours autoritaire. Ainsi du député et conseiller municipal Charles Floquet s'exprimant dans une réunion publique devant ses électeurs du $11^{\mathrm{e}}$ arrondissement de Paris, le 17 novembre 1879 :

Je ne connais quant à moi qu'une seule République [...] Je le répète, je ne connais et vous ne devez connaître qu'une seule République, la République républicaine, la République de la démocratie, la République de la nation tout entière, la République du suffrage universel. (C'est cela! - Vive adhésion et applaudissements répétés.) Oui, citoyens, comme le disait en 1848 un grand orateur, Michel de Bourges : le suffrage universel, c'est le grand arbitre entre le capital et le travail, c'est le grand arbitre qui étudie, qui concilie, qui, au besoin, juge souverainement les contestations qui peuvent s'élever entre les intérêts divers qui s'agitent dans une société civilisée. (Bravos et applaudissements.) (Floquet, 1885, p. 249)

Ici, donc, pas de discours d'autorité, mais une coénonciation autoritaire qui prend en charge l'interdiscours dans toutes ses dimensions. D'après le sténographe présent, les réactions du public sont vives. Mais est-ce le suffrage universel qui est applaudi, ou le couple Charles Floquet/Michel de Bourges, les défenseurs du monde ouvrier de 1848, voire même l'année 1848 elle-même? Si l'orateur s'efface dans le discours d'autorité, il s'avère bien évidemment central dans le discours autoritaire. Il est donc essentiel de prendre en considération les choix discursifs : Léon Gambetta, Jules Ferry, Victor Hugo, Paul Bert choisissent le discours d'autorité à propos du suffrage universel. Une lecture permet d'y voir une manipulation subtile qui clôt les débats. Mais une autre permet d'y voir la possibilité de laisser ouvert un horizon d'attente, un champ d'expérimentation d'une interaction entre citoyens réfléchissant leur expérience, constitutive du sens commun fondateur de la République.

\section{Corpus}

BERT Paul, 1881, Leçons, discours et conférences, Paris, Charpentier.

BLANC Louis, 1882, Discours politiques, 1847-1881, Paris, Germer-Baillière.

Discours et opinions de Jules Ferry, 1893, P. Robiquet éd., Paris, Armand Colin (7 volumes).

Discours et plaidoyers politiques de Gambetta, 1881, J. Reinach éd., Paris, Charpentier (11 volumes).

Floquet Charles, 1885, Discours et opinions, rassemblés par Albert Faivre, Paris, Derveaux (2 volumes).

Hugo Victor, 1968, Actes et Paroles, Fuvre complète de Victor Hugo présentée par JeanLouis Cornuz, Paris, Rencontre.

SPULLER Eugène, 1879 et 1881, Conférences populaires, première série, Paris, Dreyfous, 1879, deuxième série, Paris, Charpentier, 1881. 


\section{Références}

Amossy Ruth, 2000, L'argumentation dans le discours. Discours politique, littérature d'idées, fiction, Paris, Nathan.

Blais Marie-Claude, 2000, Au principe de la République. Le cas Renouvier, Paris, Gallimard.

Bon homme Marc, 2005, Pragmatique des figures du discours, Paris, Champion.

Constantin de Chanay Hugues, Rémi-Giraud Sylviane, 2007, "Démocratie et ses dérivés. De la dénomination à l'argument sans réplique? », Mots. Les langages du politique, no83, p. 81-99.

CosSART Paula, 2011, Le meeting politique de la délibération à la manifestation (18681939), Rennes, Presses universitaires de Rennes.

DAYAN Daniel, 1992, "Les mystères de la réception», Le Débat, nº71, septembreoctobre, Paris, Gallimard, p. 141-157.

DelPORTE Christian, 2009, Une histoire de la langue de bois, Paris, Champs Histoire.

Deluns-Montaud Pierre, 1897, "La philosophie de Gambetta », Revue politique et parlementaire, n⿳032, Paris, Armand Colin, p. 241-266.

Dontenwille-Gerbaud Aude, 2012, "Portée et vertus des nombres dans les discours des Pères fondateurs de la République», Mots. Les langages du politique, nº100, p. 43-56.

- à paraître, «Typologie des réactions des publics populaires républicains lors des grands meetings politiques. 1871-1882 », Actes du colloque «Être Peuple», Université de Lorraine, novembre 2012, Questions de communication, Éditions universitaires de Lorraine.

Guilhaumou Jacques, 2006, Discours et événement. L'histoire langagière des concepts, Besançon, Presses universitaires de Franche-Comté.

JAMEt Denis, Jobert Manuel éd., 2010, Empreintes de l'euphémisme. Tours et détours, Paris, L'Harmattan.

KANT Emmanuel, 1841, La religion dans les limites de la raison, trad. de l'allemand par J. Trullard, Paris, De Ladrange.

KoSELLECK Reinhart, 1990, Le Futur passé. Contribution à la sémantique des temps historiques, Paris, EHESS.

Maingueneau Dominique, 2002, "Scène d'énonciation », Dictionnaire d'analyse du discours, P. Charaudeau, D. Maingueneau éd., Paris, Le Seuil, p. 515-518.

Parménie Alain, Bonnier de LA Chapelle Catherine, 1985, Histoire d'un éditeur et de ses auteurs, Paris, Albin Michel.

RABATELAlain, 2004, "L'effacement énonciatif dans les discours rapportés et ses effets pragmatiques», Langages, nº156, p. 3-17.

RosanVallon Pierre, 1992, Le sacre du citoyen. Histoire du suffrage universel en France, Paris, Gallimard.

SCHEPENS Philippe, 2011, «Le concept d'idéologie analysé depuis une position phénoménologique », Semen, nº 30, p.17-41. 